\title{
Association Study between Novel CYP26 Polymorphisms and the Risk of Betel Quid-Related Malignant Oral Disorders
}

\author{
Shyh-Jong Wu, ${ }^{1}$ Yun-Ju Chen, ${ }^{2,3}$ Tien-Yu Shieh, ${ }^{4}$ Chun-Ming Chen, ${ }^{5,6}$ Yen-Yun Wang, \\ Kun-Tsung Lee, ${ }^{8}$ Yueh-Ming Lin, ${ }^{9}$ Pei-Hsuan Chien, ${ }^{3}$ and Ping-Ho Chen ${ }^{5}$ \\ ${ }^{1}$ Department of Medical Laboratory Science and Biotechnology, Kaohsiung Medical University, No. 100, \\ Shih-Chuan 1st Road, Kaohsiung 80708, Taiwan \\ ${ }^{2}$ Department of Biological Science \& Technology, I-Shou University, No. 1, Section 1, Syuecheng Road, Kaohsiung 84001, Taiwan \\ ${ }^{3}$ Department of Medical Research, E-Da Hospital, No. 1, Yida Road, Kaohsiung 82445, Taiwan \\ ${ }^{4}$ School of Oral Hygiene, Taipei Medical University, No. 250, Wuxing Street, Taipei 11031, Taiwan \\ ${ }^{5}$ School of Dentistry, College of Dental Medicine, Kaohsiung Medical University, No. 100, Shih-Chuan 1st Road, Kaohsiung 807, Taiwan \\ ${ }^{6}$ Department of Oral and Maxillofacial Surgery, Kaohsiung Medical University Hospital, Kaohsiung Medical University, \\ No. 100, Shih-Chuan 1st Road, Kaohsiung 80708, Taiwan \\ ${ }^{7}$ Department of Clinical Research, Kaohsiung Medical University Hospital, No. 100, Shih-Chuan 1st Road, Kaohsiung 80708, Taiwan \\ ${ }^{8}$ Department of Oral Hygiene, College of Dental Medicine, Kaohsiung Medical University, No. 100, Shih-Chuan 1st Road, \\ Kaohsiung 80708, Taiwan \\ ${ }^{9}$ Division of Colorectal Surgery, Department of Surgery, Kaohsiung Chang Gung Memorial Hospital and \\ Chang Gung University College of Medicine, No. 123, Dapi Road, Kaohsiung 83301, Taiwan
}

Correspondence should be addressed to Ping-Ho Chen; phchen@kmu.edu.tw

Received 13 June 2014; Accepted 26 June 2014

Academic Editor: Li-Yeh Chuang

Copyright $($ Shyh-Jong Wu et al. This is an open access article distributed under the Creative Commons Attribution License, which permits unrestricted use, distribution, and reproduction in any medium, provided the original work is properly cited.

BQ chewing may produce significant amounts of reactive oxygen species (ROS), resulting in oral mucosa damage, and ROS may be metabolized by CYP26 families. Because the CYP26 polymorphisms associated with malignant oral disorders are not well known, we conducted an association study on the associations between the single nucleotide polymorphisms (SNP) of CYP26 families and the risks of malignant oral disorders. BQ chewers with the CYP26A1 rs4411227 C/C+C/G genotype and C allele showed an increased risk of oral and pharyngeal cancer (adjusted odds ratio $(\mathrm{aOR})=2.30$ and 1.93 , respectively). The CYP26B1 rs3768647 G allele may be associated with oral and pharyngeal cancer $(\mathrm{aOR}=3.12)$ and OPMDs $(\mathrm{aOR}=2.23)$. Subjects with the rs9309462 CT genotype and $\mathrm{C}$ allele had an increased risk of oral and pharyngeal cancer $(\mathrm{aOR}=9.24$ and 8.86 , respectively) and OPMDs $(\mathrm{aOR}=8.17$ and 7.87, respectively). The analysis of joint effects between the CYP26A1 rs4411227 and CYP26B1 rs3768647/rs9309462 polymorphisms revealed statistical significance ( $\mathrm{OOR}=29.91$ and 10.03 , respectively). Additionally, we observed a significant mRNA expression of CY26A1 and CYP26B1 in cancerous tissues compared with adjacent noncancerous tissues. Our findings suggest that novel CYP26 polymorphisms are associated with an increased risk of malignant oral disorders, particularly among BQ chewers.

\section{Introduction}

Approximately 600 million people chew betel quid (BQ) in the world [1], primarily in South/Southeast Asia and the South Pacific islands [2]. BQ without tobacco is an addictive and psychostimulant substance and is a group I human carcinogen, as stated in an evaluation by the International Agency for Research on Cancer (IARC) [3, 4]. Additionally, areca nut $(\mathrm{AN})$ is the primary component in $\mathrm{BQ}$, which has also been categorized as a group I human carcinogen by the IARC [4]. BQ usage is increasingly recognized for its association with malignant oral disorders [4-10]. The malignant oral disorders include oral potentially malignant disorders (OPMDs) (i.e., oral submucous fibrosis (OSF), leukoplakia, erythroplakia, and lichen planus) and cancers of the oral cavity and pharynx. Epidemiological studies have indicated that 
BQ chewing can elevate the risk of malignant oral diseases [4-10]. A recent study found that the percentage of male BQ chewers was more than $85 \%$ among oral cancer patients [9].

A previous study suggested that chewing BQ may produce significant reactive oxygen species (ROS), such as the hydroxyl radical, which may induce the oxidative damage of oral tissue [11]. ROS are capable of inducing nucleotide modification and the generation of DNA double stranded breaks [12] and cellular 8-hydroxy-2'-deoxyguanosine (8$\mathrm{OH}-\mathrm{dG}$ ) induced DNA oxidative damage [13]. In granulocyte-differentiated HL60 cells, a previous report indicated that all-trans retinoid acid (at-RA) induces NADPH oxidasemediated ROS generation [14].

The cytochrome P450 (CYP) 26 family via oxidative metabolism to partially regulate intracellular RA compounds (such as the concentration of at-RA) affected the balance of retinoic acid (RA) in homeostasis as well as their related signal transduction [15]. RA is a vitamin A-activated metabolite that primarily regulates cell growth, differentiation, and apoptosis in the important mechanism of fetal development as well as adult life activities [16]. RA exhibits its cardioprotective effects by preventing cardiomyocyte apoptosis and ROS generation [17]. The at-RA can produce apoptosis [18] by inducing ROS formation in rat Sertoli cells $[18,19]$. In granulocyte-differentiated HL60 cells, at-RA produces NADPH oxidase-mediated ROS formation [14].

In this CYP26 family, there are two major isoforms, CYP26A1 and CYP26B1, that can be induced by RA [15]. RA compounds usually control their content through a precise balancing mechanism. We speculated that BQ use would change RA metabolism via the stimulation of CYP26B1 in the oral mucosa, and the metabolism of RA is crucial for the occurrence of oral cancer [9]. This in vivo regulation was primarily through CYP26A1 and CYP26B1 metabolism [20], and we speculated that this regulation may be associated with ROS. Thus, the specific aim of this association study was to investigate the role of CYP26 family (CYP26A1 and CYP26B1) single nucleotide polymorphisms (SNPs) in the risk of OPMDs and oral and pharyngeal cancers.

\section{Methods}

2.1. Participants and Data Collection. Patients with oral/pharyngeal cancers $(N=211)$ and OPMDs $(N=218)$ were identified from the Department of Oral and Maxillofacial Surgery, Kaohsiung Medical University Hospital in Taiwan, between 2006 and 2010. Healthy controls $(N=218)$ were recruited from a community oral health survey. All volunteers signed written informed consent and provided whole blood. The study was approved by the Institutional Review Board of Kaohsiung Medical University Hospital (KMUHIRB-970413, KMUH-IRB-950315, and KMUH-IRB-950094). The characteristics of the demographic variable and the status of substance use (such as alcohol, BQ, and cigarette use) were investigated by trained interviewers. Alcohol drinkers included current and former drinkers. Smokers included current and former smokers. Alcohol users, BQ chewers, and cigarette smokers were defined as alcoholic beverage consumption (irrespective of quantity) at least once per week for longer than 6 months, at least one quid of BQ chewed per day for longer than 6 months, and at least 10 cigarettes smoked per week for longer than 6 months, respectively. A cumulative lifetime BQ exposure (pack-years) was defined as the number of packs consumed multiplied by chewing years. One pack was defined as chewing 10 quids per day. Eight subjects with oral cancerous tissue and adjacent noncancerous oral tissue were collected during necessary surgery resection. These tissue specimens without chemotherapy or radiation therapy were analyzed. Informed consents was also signed by eight oral and pharyngeal patients.

2.2. DNA Extraction and Genotyping. Eight c.c. of peripheral blood was collected in tubes containing ethylenediaminetetraacetic acid (EDTA). Genomic DNA was extracted from the peripheral blood samples using the QIAamp DNA Mini Kit (Qiagen) according to the manufacturer's instructions. The extracted DNA samples were stored at $-80^{\circ} \mathrm{C}$ until examination. DNA concentrations were checked via optical density at 260-280 nm (Nano Drop ND-2000; Thermo Fisher Scientific Inc.).

Single-nucleotide polymorphisms (SNPs) of CYP26A1 and CYP26B1 were selected with minor allele frequency from a public reference database in the Chinese HapMap-CHB. SNP Genotyping was performed using a Taqman Genotyping Assay according to the manufacturer's instructions. All assays and gDNA were conducted in 384-well plates, and PCR was performed. After PCR amplification, an endpoint plate read was performed using an Applied Biosystems ViiA7 Real-Time PCR System. The Sequence Detection System (SDS) Software analyzed the fluorescence measurements made during the plate read to plot fluorescence $(\mathrm{Rn})$ values based on the signals from each well. The plotted fluorescence signals indicate which alleles are in each sample.

2.3. Real-Time qRT-PCR Analysis. The total RNA was extracted from oral cancerous tissues, as well as their adjacent noncancerous tissue using TRIzol (Invitrogen, Carlsbad, CA, USA) and the commercial protocol of the manufacturer as described [21]. Before further real-time qRT-PCR PCR analysis, each cDNA pool was prepared at $-20^{\circ} \mathrm{C}$. For realtime PCR assays, specific oligonucleotide primer pairs were purchased from Roche Universal ProbeLibrary. The reactions of real-time qRT-PCR were analyzed using the Roche LightCycler Instrument 1.5 with a LightCycler FastStart DNA

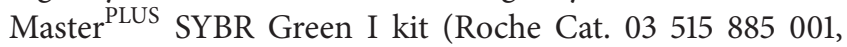
Castle Hill, Australia). The fold change of the expression of the target gene relative to the internal control gene GAPDH in each sample was calculated using the following formula: $2^{-\Delta \Delta \mathrm{Ct}}$ where $\Delta \mathrm{Ct}=\mathrm{Ct}_{\text {target gene }}-\mathrm{Ct}_{\text {internal control }}$ and $\Delta \Delta \mathrm{Ct}=$ $\Delta \mathrm{Ct}_{\text {test sample }}-\Delta \mathrm{Ct}_{\text {control sample }}$.

2.4. Statistical Analysis. The control genotype distribution complied with the Hardy-Weinberg equilibrium $(P \geq 0.05)$. In this statistical analysis, the questionnaire data included the demographic information, substance use (alcohol, betel quid, and cigarette use), and history and disease status (normal controls, OPMDs, and oral/pharynx cancer). General linear model (GLM) analysis was used for comparing the 
TABLE 1: Distribution of male betel quid chewers associated with characteristics of selected demographic factors.

\begin{tabular}{|c|c|c|c|c|}
\hline & $\begin{array}{c}\text { Oral and } \\
\text { pharyngeal } \\
\text { cancer } \\
(N=211)\end{array}$ & $\begin{array}{l}\text { OPMDs } \\
(N=56)\end{array}$ & $\begin{array}{c}\text { Control } \\
(N=218)\end{array}$ & $P$ value \\
\hline BQ chewers & $N(\%)^{\mathrm{a}}$ & $N(\%)$ & $N(\%)$ & \\
\hline Age (mean \pm S.D., years) & $50.12 \pm 9.15$ & $49.19 \pm 11.18$ & $43.57 \pm 8.58$ & $<0.05$ \\
\hline \multicolumn{5}{|l|}{ Ethnicity } \\
\hline Hokkien & $181(85.78)$ & $44(78.57)$ & $174(79.82)$ & \multirow[t]{2}{*}{0.20} \\
\hline Others & $30(14.22)$ & $12(21.43)$ & $44(20.18)$ & \\
\hline \multicolumn{5}{|l|}{ Education (years) } \\
\hline$\leq 9$ & $127(60.19)$ & $29(51.79)$ & $141(64.68)$ & \multirow[t]{2}{*}{0.19} \\
\hline$>9$ & $84(39.81)$ & $27(48.21)$ & $77(35.32)$ & \\
\hline \multicolumn{5}{|l|}{ Alcohol drinking status } \\
\hline Nondrinkers & $68(31.28)$ & $16(28.57)$ & $55(25.23)$ & \multirow[t]{2}{*}{0.38} \\
\hline Drinkers & $145(68.72)$ & $40(71.43)$ & $163(74.77)$ & \\
\hline Age at starting drinking (mean \pm S.D., years) & $22.58 \pm 6.89$ & $20.14 \pm 4.85$ & $18.60 \pm 4.73$ & $<0.05$ \\
\hline Years of alcohol drinking & $24.32 \pm 8.63$ & $26.94 \pm 10.23$ & $19.21 \pm 8.29$ & $<0.05$ \\
\hline \multicolumn{5}{|l|}{ Cigarette smoking status } \\
\hline Nonsmokers & $16(7.58)$ & $4(7.14)$ & $8(3.67)$ & \multirow[t]{2}{*}{0.20} \\
\hline Smokers & $195(92.42)$ & $52(92.86)$ & $210(96.33)$ & \\
\hline Age at starting smoking (mean \pm S.D., years) & $19.01 \pm 4.06$ & $19.35 \pm 5.08$ & $17.38 \pm 9.77$ & 0.05 \\
\hline Average amount of smoking (cigarette/day) & $26.12 \pm 14.47$ & $27.10 \pm 15.18$ & $16.06 \pm 11.03$ & $<0.05$ \\
\hline Years of cigarette smoking & $27.89 \pm 9.20$ & $28.96 \pm 9.12$ & $26.19 \pm 7.22$ & 0.05 \\
\hline \multicolumn{5}{|l|}{ BQ chewing status } \\
\hline Age at starting chewing (mean \pm S.D., years) & $22.17 \pm 6.53$ & $22.66 \pm 8.23$ & $18.99 \pm 5.07$ & $<0.05$ \\
\hline Years of BQ chewing & $21.96 \pm 8.55$ & $21.39 \pm 9.44$ & $18.60 \pm 9.16$ & $<0.05$ \\
\hline Average amount of chewing (quids/day) & $34.01 \pm 35.39$ & $34.66 \pm 27.10$ & $30.83 \pm 34.99$ & 0.59 \\
\hline Cumulative lifetime BQ use (pack-years) ${ }^{\mathrm{a}}$ & $73.91 \pm 73.58$ & $69.78 \pm 53.28$ & $58.73 \pm 72.09$ & 0.10 \\
\hline
\end{tabular}

The $P<0.05$ indicated statistical significance, and it was calculated via the Chi-square or GLM test (post hoc was compared using the Bonferroni test). Means within each row (in capital letter) followed by the different letter are statistically significant differences (via the Bonferroni test $(P<0.05)$ ).

${ }^{a}$ One chewed pack corresponds to 10 betel quids.

differences in the means between three groups, and post hoc comparisons were analyzed using the Bonferroni test. Using a multinomial logistic regression model to control for potential confounders, such as demographic factors (continuous age, ethnicity, and education levels) and substance use (cigarette and alcohol use), an exact $P$ value, adjusted odds ratio (aOR), and $95 \%$ confidence interval (CI) were produced for our tables. All statistical analyses were performed using the SAS Statistical Package (Version 9.1.3, SAS Institute Inc.).

\section{Results}

All subjects $(N=485)$ were BQ chewers. Among these, 56 OPMDs patients, 211 oral and pharyngeal cancer patients, and 218 healthy controls were recruited in this case-control study. The demographic characteristics, alcohol use status, and cigarette use status are shown in Table 1. There was statistical significance in the average ages among oral and pharyngeal cancer patients, OPMDs patients, and healthy controls $(50.12 \pm 9.15,49.19 \pm 11.18$, and $43.57 \pm 8.58$ years old, respectively). The distribution of ethnicity, education levels, alcohol drinking status, and cigarette smoking status showed no statistically significant differences. Oral and pharyngeal cancer patients exhibited significantly older age at drinking initiation compared with controls $(P<0.05)$. The oral and pharyngeal cancer and OPMDs patients showed a significantly higher average amount of smoking than the controls $(P<0.05)$. In terms of BQ chewing, we observed that oral and pharyngeal cancer and OPMDs patients had a significantly longer duration of chewing and older age at chewing initiation than the controls $(P<0.05)$. Additionally, oral and pharyngeal cancer and OPMDs patients had higher cumulative lifetime BQ use compared with the controls.

3.1. The Distribution of Genetic Polymorphisms between Oral and Pharyngeal Patients and Control Groups. Between the oral and pharyngeal patients and control groups, the difference in genotype frequency distribution was statistically significant for CYP26A1 rs4411227 (Table 2). After adjusting for covariates (age, ethnicity, education, alcohol drinking, and cigarette smoking), the results showed that BQ chewers with the rs4411227 C/G genotype or $\mathrm{C} / \mathrm{C}+\mathrm{C} / \mathrm{G}$ combined 
TABLE 2: Distribution of CYP26 families genotype and allele frequency among malignant oral disorders patients and control groups.

\begin{tabular}{|c|c|c|c|c|c|c|c|}
\hline \multirow[b]{2}{*}{ BQ chewers } & \multirow{2}{*}{$\begin{array}{c}\text { Oral and } \\
\text { pharyngeal } \\
\text { cancer } \\
(N=211) \\
N(\%)\end{array}$} & \multirow{2}{*}{$\begin{array}{c}\text { OPMDs } \\
(N=56) \\
N(\%)\end{array}$} & \multirow{2}{*}{$\begin{array}{c}\text { Controls } \\
\begin{array}{c}N=218) \\
N(\%)\end{array}\end{array}$} & \multicolumn{2}{|c|}{ Oral and pharyngeal cancer versus controls } & \multicolumn{2}{|c|}{ OPMDs versus controls } \\
\hline & & & & $\mathrm{aOR}(95 \% \mathrm{CI})$ & $P$ & $\mathrm{aOR}(95 \% \mathrm{CI})$ & $P$ \\
\hline \multicolumn{8}{|l|}{ CYP26A1 } \\
\hline \multicolumn{8}{|l|}{ rs4411227 } \\
\hline \multicolumn{8}{|l|}{ Genotype } \\
\hline $\mathrm{G} / \mathrm{G}$ & $130(61.61)$ & $40(71.43)$ & $170(77.98)$ & 1.00 & & 1.00 & \\
\hline $\mathrm{C} / \mathrm{G}$ & $74(35.07)$ & $14(25.00)$ & $43(19.72)$ & $2.38(1.48-3.84)^{\mathrm{b} *}$ & 0.0004 & $1.39(0.67-2.90)$ & 0.3792 \\
\hline $\mathrm{C} / \mathrm{C}$ & $7(3.32)$ & $2(3.57)$ & $5(2.29)$ & $1.65(0.46-5.95)$ & 0.4417 & $0.80(0.09-7.46)$ & 0.8430 \\
\hline \multicolumn{8}{|l|}{$\begin{array}{l}\text { Combined } \\
\text { genotype }\end{array}$} \\
\hline $\mathrm{G} / \mathrm{G}$ & $130(61.61)$ & $40(71.43)$ & $170(77.98)$ & 1.00 & & 1.00 & \\
\hline $\mathrm{C} / \mathrm{C}+\mathrm{C} / \mathrm{G}$ & $81(38.39)$ & $16(28.57)$ & $48(22.02)$ & $2.30(1.45-3.64)^{*}$ & 0.0004 & $1.33(0.65-2.70)$ & 0.4389 \\
\hline \multicolumn{8}{|l|}{ Allele } \\
\hline G & $334(79.15)$ & $94(83.93)$ & $383(87.84)$ & 1.00 & & 1.00 & \\
\hline $\mathrm{C}$ & $88(20.85)$ & $18(16.07)$ & $53(12.16)$ & $1.93(1.30-2.88)^{*}$ & 0.0012 & $1.22(0.65-2.29)$ & 0.5447 \\
\hline \multicolumn{8}{|l|}{ CYP26B1 } \\
\hline \multicolumn{8}{|l|}{ rs887844 } \\
\hline \multicolumn{8}{|l|}{ Genotype } \\
\hline $\mathrm{G} / \mathrm{G}$ & $115(54.50)$ & $25(44.64)$ & $133(61.01)$ & 1.00 & & 1.00 & \\
\hline $\mathrm{A} / \mathrm{G}$ & $96(45.50)$ & $31(55.36)$ & 85 (38.99) & $1.38(0.91-2.09)$ & 0.1273 & $1.87(1.01-3.49)^{*}$ & 0.0482 \\
\hline \multicolumn{8}{|l|}{ Allele } \\
\hline G & $326(77.25)$ & $81(72.32)$ & $351(80.50)$ & 1.00 & & 1.00 & \\
\hline A & $96(22.75)$ & $31(27.68)$ & $85(19.50)$ & $1.26(0.89-1.80)$ & 0.1941 & $1.55(0.93-2.57)$ & 0.0898 \\
\hline \multicolumn{8}{|l|}{ rs3768647 } \\
\hline $\mathrm{C} / \mathrm{G}$ & $103(48.82)$ & $34(60.71)$ & $218(100.00)$ & 1.00 & & 1.00 & \\
\hline $\mathrm{G} / \mathrm{G}$ & $108(51.18)$ & $22(39.29)$ & $0(0.00)$ & $-{ }^{a}$ & & $-{ }^{a}$ & \\
\hline \multicolumn{8}{|l|}{ Allele } \\
\hline $\mathrm{C}$ & $103(24.41)$ & $34(30.36)$ & $218(50.00)$ & 1.00 & & 1.00 & \\
\hline G & $319(75.59)$ & $78(69.64)$ & $218(50.00)$ & $3.12(2.28-4.27)^{*}$ & $<0.0001$ & $2.23(1.40-3.54)^{*}$ & 0.0007 \\
\hline \multicolumn{8}{|l|}{ rs9309462 } \\
\hline $\mathrm{T} / \mathrm{T}$ & $198(93.84)$ & $52(92.86)$ & $216(99.08)$ & 1.00 & & 1.00 & \\
\hline $\mathrm{C} / \mathrm{T}$ & $13(6.16)$ & $4(7.14)$ & $2(0.92)$ & $\begin{array}{c}9.24 \\
(1.90-45.00)^{*}\end{array}$ & 0.0059 & $8.17(1.25-53.52)^{*}$ & 0.0285 \\
\hline \multicolumn{8}{|l|}{ Allele } \\
\hline $\mathrm{T}$ & 409 (96.92) & $108(96.43)$ & $434(99.54)$ & 1.00 & & 1.00 & \\
\hline $\mathrm{C}$ & $13(3.08)$ & $4(3.57)$ & $2(0.46)$ & $\begin{array}{c}8.86 \\
(1.84-42.59)^{*}\end{array}$ & 0.0065 & $7.87(1.22-50.54)^{*}$ & 0.0298 \\
\hline
\end{tabular}

aOR was adjusted by continuous age, ethnicity, education level, alcohol drinking, and cigarette smoking habits.

${ }^{\mathrm{a}}$ Nonestimated: because the number of samples is equal to zero.

${ }^{\mathrm{b} *} P<0.05$.

genotype had an approximately 2 -fold increased risk for the development of oral and pharyngeal cancer relative to those with the G/G genotype. The subjects with the $\mathrm{C}$ allele had a significantly higher risk of oral and pharyngeal cancer than those in the control groups $(\mathrm{aOR}=1.93 ; 95 \% \mathrm{CI}=$ $1.30-2.88$ ). There were no significant differences in the genotype distribution of CYP26B1 rs887844 between the oral and pharyngeal patients and the controls ( $P$ values $>0.05)$.
Compared with the control groups, the subjects with the rs3768647 G allele had a significantly higher independent risk for oral and pharyngeal cancer $(\mathrm{aOR}=3.12 ; 95 \% \mathrm{CI}=$ 2.28-4.27). After adjusting for covariates, the subjects with the rs9309462 $\mathrm{C} / \mathrm{T}$ genotype $(\mathrm{aOR}=9.24 ; 95 \% \mathrm{CI}=1.90-$ 45.00) or $\mathrm{C}$ allele $(\mathrm{aOR}=8.86 ; 95 \% \mathrm{CI}=1.84-42.59)$ had a significantly higher independent risk for oral and pharyngeal cancer compared with the control groups. 
TABLE 3: Joint effects between CYP26A1 and CYP26B1 polymorphisms among malignant oral disorders patients and control groups.

\begin{tabular}{|c|c|c|c|c|c|c|}
\hline & & $\begin{array}{c}\text { Oral and } \\
\text { pharyngeal } \\
\text { cancer } \\
(N=211)\end{array}$ & $\begin{array}{l}\text { OPMDs } \\
(N=56)\end{array}$ & $\begin{array}{l}\text { Controls } \\
(N=218)\end{array}$ & $\begin{array}{c}\text { Oral and } \\
\text { pharyngeal cancer } \\
\text { versus controls }\end{array}$ & $\begin{array}{l}\text { OPMDs versus } \\
\text { controls }\end{array}$ \\
\hline BQ chewers & & $N(\%)$ & $N(\%)$ & $N(\%)$ & aOR (95\% CI) & aOR (95\% CI) \\
\hline CYP26A1 rs4411227 & CYP26B1 rs887844 & & & & & \\
\hline Allele & Allele & & & & & \\
\hline G & G & $272(64.45)$ & $75(66.96)$ & $298(68.35)$ & 1.00 & \\
\hline $\mathrm{C}$ & G & $54(12.80)$ & $6(5.36)$ & $53(12.16)$ & $1.13(0.72-1.76)$ & $0.39(0.15-1.02)$ \\
\hline G & $\mathrm{A}$ & $62(14.69)$ & $19(16.96)$ & $85(19.50)$ & $0.83(0.56-1.23)$ & $0.90(0.50-1.62)$ \\
\hline $\mathrm{C}$ & $\mathrm{A}$ & $34(8.06)$ & $12(10.71)$ & $0(0.00)$ & $-^{\mathrm{a}}$ & $-^{\mathrm{a}}$ \\
\hline CYP26A1 rs4411227 & CYP26B1 rs3768647 & & & & & \\
\hline Allele & Allele & & & & & \\
\hline G & $\mathrm{C}$ & $62(14.69)$ & $26(23.21)$ & $170(38.99)$ & 1.00 & 1.00 \\
\hline $\mathrm{C}$ & $\mathrm{C}$ & $41(9.72)$ & $8(7.14)$ & 48 (11.01) & $2.51(1.45-4.34)^{\mathrm{b} *}$ & $1.17(0.48-2.82)$ \\
\hline G & G & $272(64.45)$ & $68(60.71)$ & $213(48.85)$ & $3.64(2.51-5.26)^{*}$ & $2.14(1.28-3.59)^{*}$ \\
\hline $\mathrm{C}$ & G & 47 (11.14) & $10(8.93)$ & $5(1.15)$ & $\begin{array}{c}29.91 \\
(10.75-83.23)^{*}\end{array}$ & $11.25(3.18-39.77)^{*}$ \\
\hline CYP26A1 rs4411227 & CYP26B1 rs9309462 & & & & & \\
\hline Allele & Allele & & & & & \\
\hline G & $\mathrm{T}$ & $327(77.49)$ & $92(82.14)$ & $382(87.61)$ & 1.00 & 1.00 \\
\hline $\mathrm{C}$ & $\mathrm{T}$ & $82(19.43)$ & $16(14.29)$ & $52(11.93)$ & $1.85(1.23-2.77)^{*}$ & $1.07(0.55-2.08)$ \\
\hline G & $\mathrm{C}$ & $7(1.66)$ & $2(1.79)$ & $1(0.23)$ & $9.44(1.08-82.43)^{*}$ & $4.58(0.27-77.85)$ \\
\hline $\mathrm{C}$ & $\mathrm{C}$ & $6(1.42)$ & $2(1.79)$ & $1(0.23)$ & $10.03(1.05-95.60)^{*}$ & $12.20(0.99-151.07)$ \\
\hline
\end{tabular}

aOR was adjusted by continuous age, ethnicity, education level, alcohol drinking, and cigarette smoking habits.

${ }^{a}$ Nonestimated: because the number of samples is equal to zero.

${ }^{\mathrm{b} *} P<0.05$

3.2. The Distribution of Genetic Polymorphisms between the OPMDs Patients and Control Groups. There were no differences in rs4411227 polymorphism distribution between the OPMDs patients and controls (Table 2). After adjusting the covariates (age, ethnicity, education, alcohol drinking, and cigarette smoking), the subjects carrying the rs887844 A/G genotype had a marginally enhanced risk for OPMDs ( $\mathrm{aOR}=$ 1.87; 95\% CI $=1.01-3.49 ; P=0.0482$ ) compared with the G/G type. Individuals with the rs3768647 G allele had a 2.23fold greater risk of OPMDs compared with those with the $\mathrm{C}$ allele $(\mathrm{aOR}=2.23 ; 95 \% \mathrm{CI}=1.40-3.54)$. The subjects with the rs9309462 C/T genotype and $\mathrm{C}$ allele showed a significantly higher risk compared with the subjects with the $\mathrm{TT}$ and $\mathrm{T}$ allele genotype ( $\mathrm{aOR}=8.17 ; 7.87$, respectively).

3.3. The Gene-Gene Joint Effects in the Risk of Oral and Pharyngeal Cancer and OPMDs. We further analyzed the gene-gene joint effects in the risk of oral and pharyngeal cancer and OPMDs (Table 3), after adjusting the covariates (age, ethnicity, education, alcohol drinking, and cigarette smoking). BQ chewers with the CYP26A1 rs4411227 C allele and CYP26B1 rs3768647 $\mathrm{G}$ allele had the highest risk of oral and pharyngeal cancer compared with the subjects carrying the CYP26A1 rs4411227 G allele and CYP26B1 rs3768647 C allele $(\mathrm{aOR}=29.91 ; 95 \% \mathrm{CI}=10.75-83.23)$. Similarly, individuals carrying the CYP26A1 rs4411227 C allele and
CYP26B1 rs3768647 G allele had the highest risk of OPMDs relative to the subjects carrying the CYP26A1 rs4411227 G allele and CYP26B1 rs3768647 $\mathrm{C}$ allele $(\mathrm{aOR}=11.25$; 95\% CI $=3.18-39.77)$. BQ chewers with the CYP26A1 rs4411227 C allele and CYP26B1 rs9309462 C allele had the highest risk of oral and pharyngeal cancer compared with the subjects carrying the CYP26A1 rs4411227 G allele and CYP26B1 rs9309462 $\mathrm{T}$ allele $(\mathrm{aOR}=10.03$; 95\% $\mathrm{CI}=$ 1.05-95.60). However, there were no significant differences on gene-gene joint effects between the presence of the CYP26A1 rs4411227 and CYP26B1 rs9309462 genetic variations regarding the risk of OPMDs.

3.4. The CYP26A1 and CYP26B1 mRNA Expression of Oral Paired Tissue and Adjacent Noncancerous Tissues. We investigated CYP26A1 and CYP26B1 quantitative mRNA in expression in eight patients (numbers $1,2,3,4,5,6,7$, and 8) (Figure 1). Compared with their adjacent noncancerous tissues, tumor tissues exhibited the consistent downregulation mRNA of CYP26A1 and CYP26B1 in patients number 2, 3, 5, and 6 (expression $>2$-fold change in numbers 2,3 , and 5). In patients number 1 and 8 , the upregulation of the expression of CYP26A1 and CYP26Blin cancerous tissue was observed, compared with their adjacent noncancerous tissues. In the cancer tissue of number 4, a slightly decreased expression of CYP26A1 and increased expression of CYP26B1 were found. 


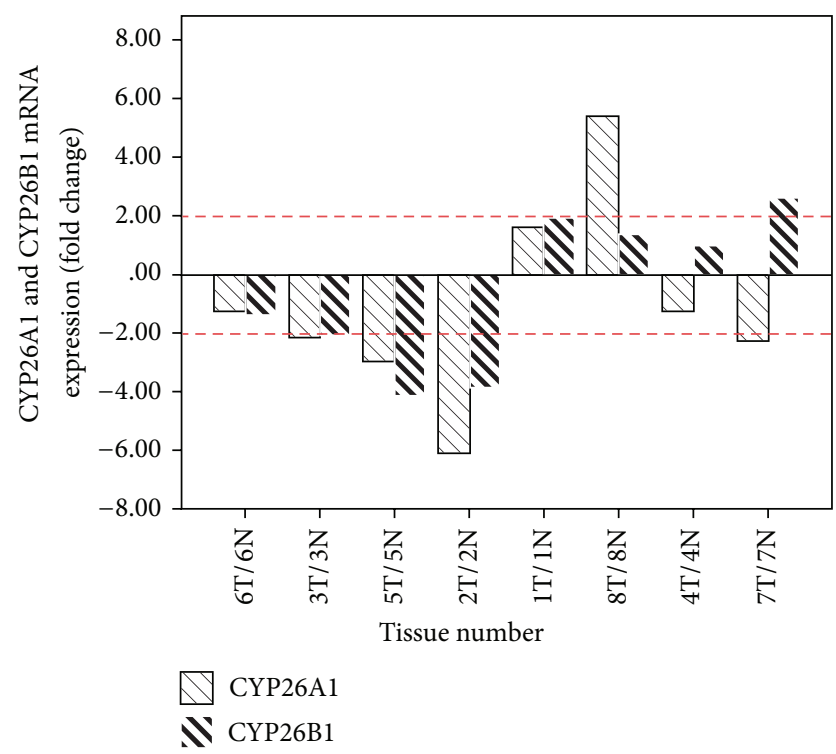

Figure 1: The induced mRNA of CYP26A1 and CYP26B1 in human oral cancer tissue (T) and its adjacent normal tissue (N). Paired tissue samples (tumor and adjacent normal tissue) without chemotherapy/radiation therapy were analyzed. Compared with human adjacent tissue $(N=8)$, the relative fold change was calculated in triplicate (columns, mean; bars, SD) using the formula $2^{-\Delta \Delta \mathrm{Ct}}$

CYP26A1 expression was lower in the cancer tissue of number 7 compared with its adjacent tissue, but the expression of CYP26B1 was higher in the cancer tissue than in the adjacent tissue.

\section{Discussion}

The International Agency for Research on Cancer (IARC) has indicated that betel quid without tobacco can cause oral cancer and has stated that, in experimental animals, there is sufficient evidence to establish the carcinogenicity of the areca nut; there is limited evidence for the carcinogenicity of arecoline [4]. The areca nut ( $\mathrm{AN})$ is a major ingredient of $B Q$, and arecoline is the most abundant AN alkaloid. In detoxifying $\mathrm{AN}$ or arecoline, two monooxygenase systems (cytochrome P450 and flavin-containing monooxygenases) are implicated in phase I metabolism [22]. A previous report indicated that the monooxygenase activity of CYP26B1 may be involved in the detoxification process of BQ chewing [22]. Furthermore, CYP26B1 has been demonstrated to participate in the metabolism of at-RA and has been indicated to play a major role in the protection of specific tissues for at-RA exposure [23]. A recent study demonstrated that the retinoic acid-metabolizing enzymes CYP26A1 and CYP26B1 are significantly overexpressed in colorectal cancer tissue [24].

This is the first study to indicate that the mRNA expression of CYP26 families (CYP26A1 and CYP26B1) and their SNP variants play a novel role in the occurrence or developmental mechanism of malignant oral disorders. In our analysis, we evaluated the risk effect of CYP polymorphisms among BQ chewers. These findings showed that BQ chewers with CYP26A1 risk polymorphism (rs4411227) may enhance the risk of oral and pharyngeal cancer. Also, subjects carrying CYP26B1 risk polymorphism (rs3768647/rs9309462) have an increased susceptibility to oral malignant disorders. The CYP26A1 rs4411227 and CYP26B1 rs3768647/rs9309462 may have significant joint effects in the risk of oral malignant disorders (particularly in oral and pharyngeal patients) among BQ chewers. To rule out differences in gene expression between different individuals, we collected paired oral tissues to observe the significant expression of CYP26A1 and CYP26B1. Overall, these findings seem to have strengths on the role of CYP26A1 and CYP26B1 in the etiology of oral malignant disorders.

4.1. The Susceptible Metabolic CYP26B1 Gene. The CYP26B1 gene is located on chromosome $2 \mathrm{p} 13.2$ and covers a total of eighteen thousand base pairs. After transcription, the CYP26B1 gene formed 6 exons and $8.57 \mathrm{~kb}$ introns and included an approximately $3 \mathrm{~kb}$ long untranslated $3^{\prime}$ region $[25,26]$. It is also a single-oxygenase enzyme (monooxygenase) that catalyzes many reactions, such as those involving drug metabolism and the synthesis of hormones, cholesterol, and lipids. However, the catalytic function of CYP26B1 can catalyze at-RA into a hydroxylated form; this also refers to the process of the oxidation of RA through the added oxygen in number 4 seat of the carbon skeleton, subsequently metabolizing RA into the polar and inactive form (such as 4-oxo-,4$\mathrm{OH}$-,5,6-epoxy and 18-OH-all-trans-retinoic acid) to activate it [27] while not affecting the cell physiology. A previous report suggested that CYP26B1 appears to be necessary in the physiological role of RA catabolism, whereas CYP26A1 played an important role given excessive RA in the cells [28].

4.2. The Susceptible Metabolic CYP26A1 Gene. CYP26A1 is located on chromosome 10q23-q24 [29]. CYP26A1 and CYP26B1 are at-RA hydroxylases that are responsible for the catalytic formation of similar metabolites in a cellular system; there is only $40 \%$ similarity among the CYP26A1 gene sequence and CYP26B1 gene sequence [30]. CYP26A1 is a hydroxylation enzyme for the major metabolism of RA and transforms RA into an inactive RA hydroxy derivative $[31,32]$. CYP26A1 has high specificity for at-RA and oxidation $\mathrm{RA}$ to form 4-OH-RA, 18-OH-RA, and 4-oxo-RA [33]. Scholars found that CYP26A1 had a higher catalytic ability compared with CYP26B1 and that CYP26A1 was primarily responsible for the metabolism of at-RA and provides a protective barrier to avoid at-RA overexposure [30]. CYP26A1 may be associated with the metabolism of RA in human epidermal keratinocytes [28]. A previous report indicated that, in long term sunlight-damaged skin cells and in the increased expression of RA-metabolizing enzymes, CYP26A1 may cause a deficiency of vitamin A, which could potentially lead to the malignant transformation of keratinocytes in the early development of skin cancer [34].

A review article indicated that the inhibition of CYP26A1 expression reduces tumorigenicity through the use of RA metabolism blocking agents (RAMBAs) [15]. Previous studies demonstrated that an increased expression of CYP26A1 was found in human familial adenomatous polyposis adenomas, 
sporadic colon cancers, and primary ovarian cancer [20, 35]. A report noted that RA can induce CYP26A1 expression in neuroblastoma, breast cancer, and lung cancer cell lines [36]. In breast cancer or colon cancer cells, CYP26A1 gene expression can be induced via the receptor of vitamin A [37]. In breast epithelial adenocarcinoma tissue cultures, head and neck squamous cell carcinoma cells, and acute promyelocytic leukemia (acute promyelocytic leukemia) cells, an increased expression of CYP26A1 and increased catabolic activity of RA can be detected [38-40].

Additionally, 42\% (27/65) of tissue samples removed from breast cancer patients had CYP26A1 overexpression; CYP26A1 overexpression may induce intracellular RA consumption, thus pushing the cells toward tumorigenicity; CYP26A1 may be recommended as a candidate oncogene [41]. Researchers found that some CYP450 genes (e.g., CYP26A1) in primary ovarian cancer have significantly higher expressions compared with normal ovarian tissues [35]. Additionally, CYP26A1 overexpression in Barrett's esophagus adenocarcinoma may cause the consumption of intracellular vitamin A acid [42], whereas other reports found that the expression of CYP26A1 is lower in normal human epidermal cells $[43,44]$. After RA treatment, at-RA turnover rates are approximately 18 -fold higher in squamous head and neck cancer cell lines compared with normal oral keratinocytes; 4-oxo-RA and 4-hydroxy-RA are also generated in the former. Two squamous head and neck cancer cell lines have increased expressions of CYP26 A1 mRNA and showed the highest metabolism of RA [33]. In head and neck cancer patients, the adjacent normal oral keratinocytes showed a 15fold higher normal oral keratinocyte turnover rate compared with noncancer patients [45]. The above results suggest that RA metabolism potentially played a role in the development of oral cancer. Similarly, recent studies indicated that the increased expression of CYP26A1 genes was related to head and neck cancer [33]. This study also suggested that increasing concentrations of endogenous RA and CYP26A1 inhibitors will be applied in the future treatment of cancer or novel therapies for skin diseases $[15,46]$.

4.3. Retinoic Acid (RA) and Cancer Development. Previous studies reported that the damage of normal RA homeostasis signaling was associated with the development of cancer [47]. The damage to normal RA signaling may be due to the decreased expression of the RA receptor, the decreased transcriptional response of the RA target gene, and increased RA metabolism [48]. Since 1920, vitamin A deficiency has been associated with cell carcinogenesis [49]. Vitamin A deficiency may be associated with increased susceptibility to cancer, and low amounts of vitamin A intake may increase the risk of human cancer [50].

Research data indicated that increased RA compound intake can reduce different varieties of squamous cell carcinoma (such as oral cancer, lung cancer, pharynx cancer, cervical cancer, and bladder cancer); therefore, the deficiency of RA ingestion may result in excessive cell proliferation (hyperplasia) and hyperkeratosis and cause carcinogenesis in oral cavity cells [51]. RA compounds and their isomers can be applied to treat or prevent cancer and skin diseases [48].
A series of intervention studies has indicated that vitamin A can effectively reduce the remission of betel chewers with oral leukoplakia and that it suppressed the occurrence of new oral lesions [52-54]. Previous studies recommended the use of RA treatment for the remission of malignant transformation among BQ chewers with OPMDs; this mechanism may be due to the inhibition of BQ compounds promoting carcinogenesis rather than to them inhibiting the initiation of carcinogenesis development [52, 54, 55]. Additionally, a review article suggested that the inhibition of CYP26 enzyme activity could help to increase the half-life of RA and would be clinically effective for future applications.

CYP polymorphisms related ROS provide important insight into the importance of clinical diagnostic tools (e.g., screen test of SNP) in BQ chewers for the prevention of malignant oral disorders [56]. The study limitations were a smaller sample size for evaluation of mRNA expression and lack of phenotype information among patients. In the future research, we will collect sufficient samples to confirm expression of CYP26A1 and CYP26 B1 in betel quid-related malignant oral disorders.

In conclusion, this study suggested that BQ chewers with ROS related to CYP26A1 and CYP26B1 polymorphisms are associated with an increased risk of oral and pharyngeal cancer and OPMDs. Our findings may be useful in identifying subjects who are at an increased risk for the development of oral malignant disorders.

\section{Conflict of Interests}

The authors declare that there is no conflict of interests regarding the publication of this paper.

\section{Authors' Contribution}

Shyh-Jong Wu and Yun-Ju Chen equally contributed to this work.

\section{Acknowledgments}

This study was supported by Chi-Mei Medical Center and Kaohsiung Medical University Research Foundation Grants (100CM-KMU-13 and 101CM-KMU-12), a NSC Grant from the Ministry of Science and Technology Grant (NSC 102-2314-B-037-073-), I-Shou University Grant (ISU-103-0407A), and the Health and Welfare Surcharge of Tobacco Products Grant, the Ministry of Health and Welfare, Taiwan (MOHW103-TD-B-111-05).

\section{References}

[1] B. J. Boucher and N. Mannan, "Metabolic effects of the consumption of Areca catechu," Addiction Biology, vol. 7, no. 1, pp. 103-110, 2002.

[2] P. C. Gupta and S. Warnakulasuriya, "Global epidemiology of areca nut usage," Addiction Biology, vol. 7, no. 1, pp. 77-83, 2002.

[3] A. R. Winstock, C. R. Trivedy, K. A. A. S. Warnakulasuriya, and T. J. Peters, "A dependency syndrome related to areca nut use: some medical and psychological aspects among areca nut users 
in the Gujarat community in the UK," Addiction Biology, vol. 5, no. 2, pp. 173-179, 2000.

[4] IARC, Betel-Quid and Areca-Nut Chewing and Some ArecaNut-Derived Nitrosamines, vol. 85 of IARC Monographs on the Evaluation of Carcinogenic Risks to Humans, 2004.

[5] Y. C. Ko, Y. L. Huang, C. H. Lee et al., "Betel quid chewing, cigarette smoking and alcohol consumption related to oral cancer in Taiwan," Journal of Oral Pathology and Medicine, vol. 24, no. 10, pp. 450-453, 1995.

[6] C. H. Lee, Y. C. Ko, H. L. Huang et al., "The precancer risk of betel quid chewing, tobacco use and alcohol consumption in oral leukoplakia and oral submucous fibrosis in southern Taiwan," The British Journal of Cancer, vol. 88, no. 3, pp. 366372, 2003.

[7] K. W. Lee, W. R. Kuo, S. M. Tsai et al., "Different impact from betel quid, alcohol and cigarette: risk factors for pharyngeal and laryngeal cancer," International Journal of Cancer, vol. 117, no. 5, pp. 831-836, 2005.

[8] S. L. Chiang, P. H. Chen, C. H. Lee et al., "Up-regulation of inflammatory signalings by areca nut extract and role of cyclooxygenase-2 -119G>a polymorphism reveal risk of oral cancer," Cancer Research, vol. 68, no. 20, pp. 8489-8498, 2008.

[9] P. H. Chen, K. W. Lee, C. H. Chen et al., "CYP26B1 is a novel candidate gene for betel quid-related oral squamous cell carcinoma," Oral Oncology, vol. 47, no. 7, pp. 594-600, 2011.

[10] C. Y. Yen, S. Y. Liu, C. H. Chen et al., "Combinational polymorphisms of four DNA repair genes XRCC1, XRCC2, XRCC3, and XRCC4 and their association with oral cancer in Taiwan," Journal of Oral Pathology and Medicine, vol. 37, no. 5, pp. 271$277,2008$.

[11] P. H. Chen, C. C. Tsai, Y. C. Lin et al., "Ingredients contribute to variation in production of reactive oxygen species by areca quid," Journal of Toxicology and Environmental Health A: Current Issues, vol. 69, no. 11, pp. 1055-1069, 2006.

[12] C. C. Chiu, J. W. Haung, F. R. Chang et al., "Golden berryderived $4 \beta$-hydroxywithanolide e for selectively killing oral cancer cells by generating ROS, DNA damage, and apoptotic pathways," PLoS ONE, vol. 8, no. 5, Article ID e64739, 2013.

[13] T. Y. Liu, C. L. Chen, and C. W. Chi, "Oxidative damage to DNA induced by areca nut extract," Mutation Research, vol. 367, no. 1, pp. 25-31, 1996.

[14] C. Y. Wang, T. T. Yang, C. L. Chen, W. C. Lin, and C. F. Lin, "Reactive oxygen species-regulated glycogen synthase kinase3beta activation contributes to all-trans retinoic acid-induced apoptosis in granulocyte-differentiated HL60 cells," Biochemical Pharmacology, vol. 88, no. 1, pp. 86-94, 2014.

[15] A. C. Ross and R. Zolfaghari, "Cytochrome P450s in the regulation of cellular retinoic acid metabolism," Annual Review of Nutrition, vol. 31, pp. 65-87, 2011.

[16] T. R. J. Evans and S. B. Kaye, "Retinoids: present role and future potential," British Journal of Cancer, vol. 80, no. 1-2, pp. 1-8, 1999.

[17] R. S. Guleria, R. Choudhary, T. Tanaka, K. M. Baker, and J. Pan, "Retinoic acid receptor-mediated signaling protects cardiomyocytes from hyperglycemia induced apoptosis: role of the renin-angiotensin system," Journal of Cellular Physiology, vol. 226, no. 5, pp. 1292-1307, 2011.

[18] M. L. Conte da Frota Jr., E. Gomes da Silva, G. A. Behr et al., "Alltrans retinoic acid induces free radical generation and modulate antioxidant enzyme activities in rat sertoli cells," Molecular and Cellular Biochemistry, vol. 285, no. 1-2, pp. 173-179, 2006.
[19] N. Hail Jr., H. J. Kim, and R. Lotan, "Mechanisms of fenretinideinduced apoptosis," Apoptosis, vol. 11, no. 10, pp. 1677-1694, 2006.

[20] D. N. Shelton, I. T. Sandoval, A. Eisinger et al., "Up-regulation of CYP26A1 in adenomatous polyposis coli-deficient vertebrates via a WNT-dependent mechanism: implications for intestinal cell differentiation and colon tumor development," Cancer Research, vol. 66, no. 15, pp. 7571-7577, 2006.

[21] C. Y. Yen, C. H. Chen, C. H. Chang et al., "Matrix metalloproteinases (MMP) 1 and MMP10 but not MMP12 are potential oral cancer markers," Biomarkers, vol. 14, no. 4, pp. 244-249, 2009.

[22] S. L. Chiang, S. S. Jiang, Y. J. Wang et al., "Characterization of arecoline-induced effects on cytotoxicity in normal human gingival fibroblasts by global gene expression profiling," Toxicological Sciences, vol. 100, no. 1, pp. 66-74, 2007.

[23] J. A. White, H. Ramshaw, M. Taimi et al., "Identification of the human cytochrome P450, P450RAI-2, which is predominantly expressed in the adult cerebellum and is responsible for alltrans-retinoic acid metabolism," Proceedings of the National Academy of Sciences of the United States of America, vol. 97, no. 12, pp. 6403-6408, 2000.

[24] G. T. Brown, B. G. Cash, D. Blihoghe et al., "The expression and prognostic significance of retinoic acid metabolising enzymes in colorectal cancer," PLoS ONE, vol. 9, no. 3, Article ID e90776, 2014.

[25] Y. Yamamoto, R. Zolfaghari, and A. C. Ross, "Regulation of CYP26 (cytochrome P450RAI) mRNA expression and retinoic acid metabolism by retinoids and dietary vitamin A in liver of mice and rats," The FASEB Journal, vol. 14, no. 13, pp. 2119-2127, 2000.

[26] D. R. Nelson, "A second CYP26 P450 in humans and zebrafish: CYP26B1," Archives of Biochemistry and Biophysics, vol. 371, no. 2, pp. 345-347, 1999.

[27] J. Marill, T. Cresteil, M. Lanotte, and G. G. Chabot, "Identification of human cytochrome P450s involved in the formation of all-trans-retinoic acid principal metabolites," Molecular Pharmacology, vol. 58, no. 6, pp. 1341-1348, 2000.

[28] E. Pavez Loriè, H. Li, A. Vahlquist, and H. Törmä, “The involvement of cytochrome p450 (CYP) 26 in the retinoic acid metabolism of human epidermal keratinocytes," Biochimica et Biophysica Acta-Molecular and Cell Biology of Lipids, vol. 1791, no. 3, pp. 220-228, 2009.

[29] J. A. White, B. Beckett, S. W. Scherer, J. Herbrick, and M. Petkovich, "P450RAI (CYP26A1) maps to human chromosome 10q23-q24 and mouse chromosome 19C2-3," Genomics, vol. 48, no. 2, pp. 270-272, 1998.

[30] A. R. Topletz, J. E. Thatcher, A. Zelter et al., "Comparison of the function and expression of CYP26A1 and CYP26B1, the two retinoic acid hydroxylases," Biochemical Pharmacology, vol. 83, no. 1, pp. 149-163, 2012.

[31] J. D. Lutz, V. Dixit, C. K. Yeung et al., "Expression and functional characterization of cytochrome P450 26A1, a retinoic acid hydroxylase," Biochemical Pharmacology, vol. 77, no. 2, pp. 258268, 2009.

[32] K. Niederreither, S. Abu-Abed, B. Schuhbaur, M. Petkovich, P. Chambon, and P. Dollé, "Genetic evidence that oxidative derivatives of retinoic acid are not involved in retinoid signaling during mouse development," Nature Genetics, vol. 31, no. 1, pp. 84-88, 2002.

[33] I. Klaassen, R. H. Brakenhoff, S. J. Smeets, G. B. Snow, and B. J. M. Braakhuis, "Enhanced turnover of all-trans-retinoic 
acid and increased formation of polar metabolites in head and neck squamous cell carcinoma lines compared with normal oral keratinocytes," Clinical Cancer Research, vol. 7, no. 4, pp. 10171025, 2001.

[34] M. Osanai and G.-H. Lee, "Enhanced expression of retinoic acid-metabolizing enzyme CYP26A1 in sunlight-damaged human skin," Medical Molecular Morphology, vol. 44, no. 4, pp. 200-206, 2011.

[35] D. Downie, M. C. E. McFadyen, P. H. Rooney et al., "Profiling cytochrome P450 expression in ovarian cancer: identification of prognostic markers," Clinical Cancer Research, vol. 11, no. 20, pp. 7369-7375, 2005.

[36] T. Liu, A. Bohlken, S. Kuljaca et al., "The retinoid anticancer signal: mechanisms of target gene regulation," British Journal of Cancer, vol. 93, no. 3, pp. 310-318, 2005.

[37] E. Sonneveld, C. E. van den Brink, B. M. van der Leede et al., "Human retinoic acid (RA) 4-hydroxylase (CYP26) is highly specific for all-trans-RA and can be induced through RA receptors in human breast and colon carcinoma cells," Cell Growth and Differentiation, vol. 9, no. 8, pp. 629-637, 1998.

[38] J. van Heusden, W. Wouters, F. C. S. Ramaekers et al., “The antiproliferative activity of all-trans-retinoic acid catabolites and isomers is differentially modulated by liarozole-fumarate in MCF-7 human breast cancer cells," The British Journal of Cancer, vol. 77, no. 8, pp. 1229-1235, 1998.

[39] I. Klaassen, R. H. Brakenhoff, S. J. Smeets, G. B. Snow, and B. J. M. Braakhuis, "Metabolism and growth inhibition of four retinoids in head and neck squamous normal and malignant cells," British Journal of Cancer, vol. 85, no. 4, pp. 630-635, 2001.

[40] C. R. Larsen, P. B. Hansen, and N. T. Clausen, "all-trans-retinoic acid-induced expression and regulation of retinoic acid 4hydroxylase (CYP26) in human promyelocytic leukemia," The American Journal of Hematology, vol. 70, no. 1, pp. 39-47, 2002.

[41] M. Osanai, N. Sawada, and G. H. Lee, "Oncogenic and cell survival properties of the retinoic acid metabolizing enzyme, CYP26A1," Oncogene, vol. 29, no. 8, pp. 1135-1144, 2010.

[42] C. L. Chang, E. Hong, P. Lao-Sirieix, and R. C. Fitzgerald, "A novel role for the retinoic acid-catabolizing enzyme CYP26A1 in Barrett's associated adenocarcinoma," Oncogene, vol. 27, no. 21, pp. 2951-2960, 2008.

[43] R. Heise, J. Mey, M. M. Neis et al., "Skin retinoid concentrations are modulated by CYP26AI expression restricted to basal keratinocytes in normal human skin and differentiated 3D skin models," Journal of Investigative Dermatology, vol. 126, no. 11, pp. 2473-2480, 2006.

[44] C. Popa, A. J. Dicker, A. L. Dahler, and N. A. Saunders, "Cytochrome P450, CYP26AI, is expressed at low levels in human epidermal keratinocytes and is not retinoic acid-inducible," The British Journal of Dermatology, vol. 141, no. 3, pp. 460-468, 1999.

[45] I. Klaassen, J. Cloos, S. J. Smeets et al., "Nonmalignant oral keratinocytes from patients with head and neck squamous cell carcinoma show enhanced metabolism of retinoic acid," Oncology, vol. 63, no. 1, pp. 56-63, 2002.

[46] C. H. Nelson, B. R. Buttrick, and N. Isoherranen, “Therapeutic potential of the inhibition of the retinoic acid hydroxylases CYP26A1 and CYP26B1 by xenobiotics," Current Topics in Medicinal Chemistry, vol. 13, no. 12, pp. 1402-1428, 2013.

[47] S. Y. Sun and R. Lotan, "Retinoids and their receptors in cancer development and chemoprevention," Critical Reviews in Oncology/Hematology, vol. 41, no. 1, pp. 41-55, 2002.
[48] S. J. Freemantle, M. J. Spinella, and E. Dmitrovsky, "Retinoids in cancer therapy and chemoprevention: promise meets resistance," Oncogene, vol. 22, no. 47, pp. 7305-7315, 2003.

[49] S. B. Wolbach and P. R. Howe, "Tissue changes following deprivation of fat-soluble a vitamin," The Journal of Experimental Medicine, vol. 42, no. 6, pp. 753-777, 1925.

[50] N. Wald, M. Idle, J. Boreham, and A. Bailey, "Low serumvitamin-A and subsequent risk of cancer. Preliminary results of a prospective study," The Lancet, vol. 2, no. 8199, pp. 813-815, 1980.

[51] S. Graham, "Epidemiology of retinoids and cancer," Journal of the National Cancer Institute, vol. 73, no. 6, pp. 1423-1428, 1984.

[52] H. F. Stich, A. P. Hornby, B. Mathew, R. Sankaranarayanan, and M. K. Nair, "Response of oral leukoplakias to the administration of vitamin A," Cancer Letters, vol. 40, no. 1, pp. 93-101, 1988.

[53] H. F. Stich, B. Mathew, R. Sankaranarayanan, and M. K. Nair, "Remission of oral precancerous lesions of tobacco/areca nut chewers following administration of beta-carotene or vitamin A, and maintenance of the protective effect," Cancer Detection and Prevention, vol. 15, no. 2, pp. 93-98, 1991.

[54] H. F. Stich, M. P. Rosin, A. P. Hornby, B. Mathew, R. Sankaranarayanan, and M. K. Nair, "Remission of oral leukoplakias and micronuclei in tobacco/betel quid chewers treated with betacarotene and with beta-carotene plus vitamin A," International Journal of Cancer, vol. 42, no. 2, pp. 195-199, 1988.

[55] H. F. Stich and S. S. Tsang, "Promoting activity of betel quid ingredients and their inhibition by retinol," Cancer Letters, vol. 45, no. 1, pp. 71-77, 1989.

[56] C. Y. Lin, T. S. Pan, C. C. Ting et al., "Cytochrome P450 metabolism of betel quid-derived compounds: implications for the development of prevention strategies for oral and pharyngeal cancers," The Scientific World Journal, vol. 2013, Article ID 618032, 11 pages, 2013. 

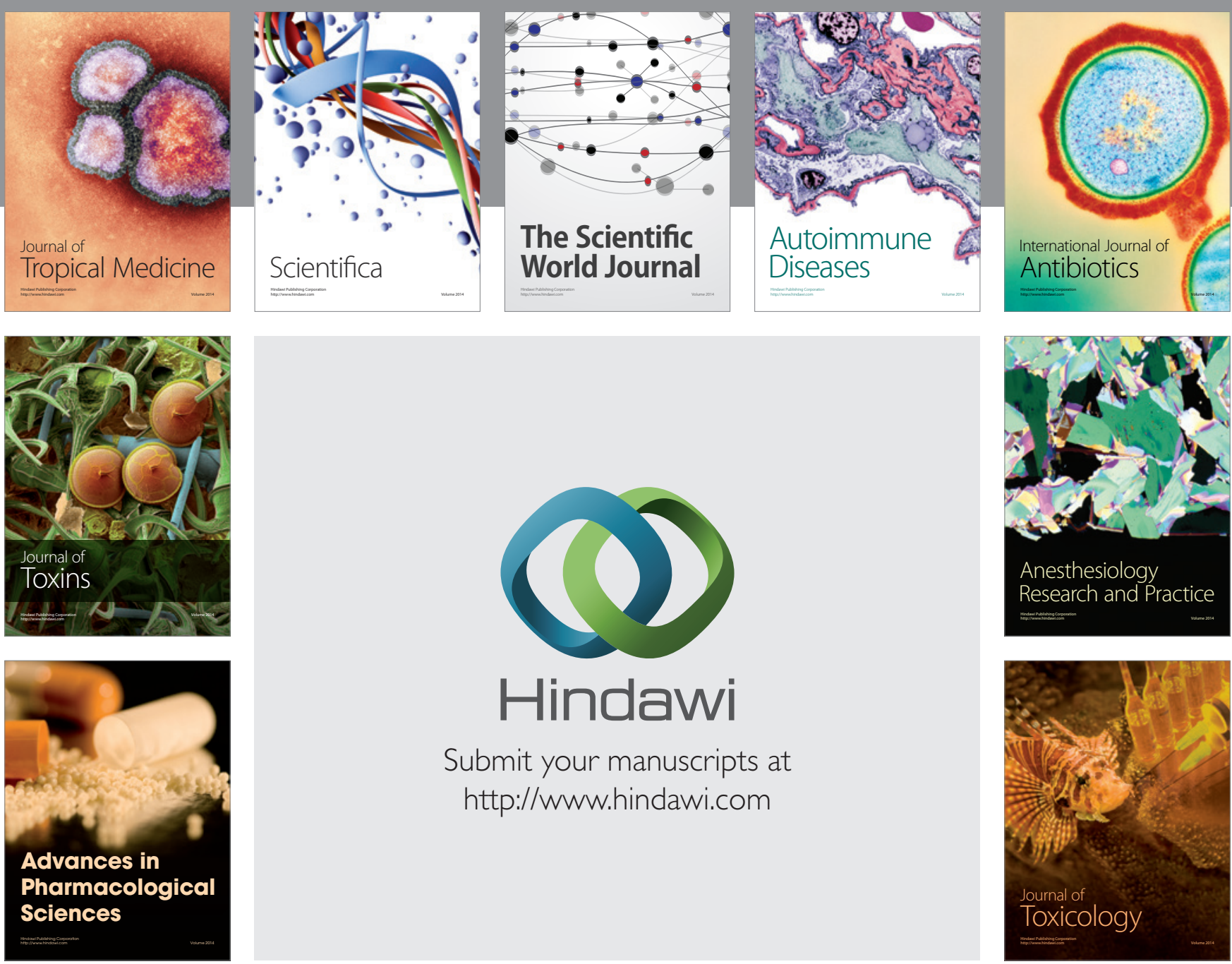

\section{Hindawi}

Submit your manuscripts at

http://www.hindawi.com
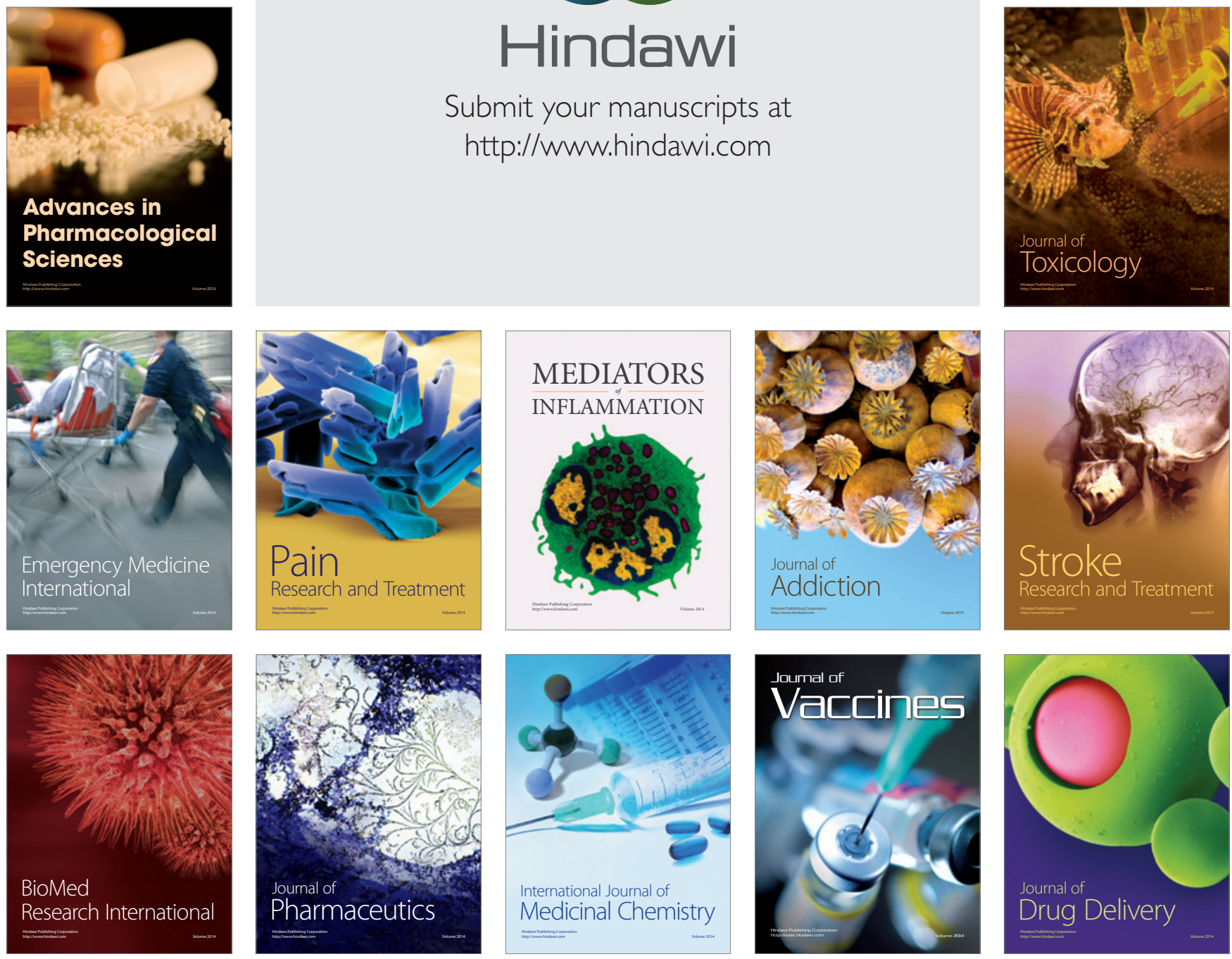